\title{
Programmable Optical Transmission Systems in the Hyperconnectivity Era: A Synergy of Photonic Technologies and Software-Defined Networking
}

\author{
Michela Svaluto Moreolo, Josep M. Fabrega and Laia Nadal \\ Centre Tecnologic de Telecomunicacions de Catalunya (CTTC/CERCA), Av. Carl Friedrich Gauss 7, 08860 Castelldefels (Barcelona), Spain. \\ michela.svaluto@cttc.es
}

\begin{abstract}
Photonic technologies and SDN are key to support hyperconnectivity in a globallynetworked society. We present transmission systems based on SDN-enabled transceivers addressing this challenge. Programmability and technological aspects are discussed.

OCIS codes: (060.4510) Optical communications; (060.4250) Networks; (250.5300) Photonic integrated circuits.
\end{abstract}

\section{Introduction}

Hyperconnectivity is a term, first used in social science, referring to person-to-person, person-to-machine and machine-to-machine interaction using multiple means of communication over the network. Timely addressing the related technological challenges is particularly relevant for the tied high societal impact. From a technological viewpoint, hyperconnectivity is driving huge capacity increase in a highly dynamic scenario enabling novel and bandwidth-hungry services, as well as internet-of-everything connectivity, to be supported by future networks [1].

A synergy of photonic technologies and software-defined networking (SDN) is crucial to successfully address the challenges posed by this evolutionary scenario in the hyperconnectivity era. Specifically, it is in the suitable design and implementation of programmable adaptive/advanced optical transmission systems, where photonic technologies and SDN meet to enhance the potential abilities and capabilities of both prominent performers.

In this context, the bandwidth/bitrate variable transceiver (BVT) represents a key element, which allows integrating in synergetic fashion novel photonic technologies with an SDN-based control plane [2]. Sliceable BVT (S-BVT) architectures able to generate multiple adaptive and variable flows, (re)-configurable via SDN agents, can be designed to have a modular implementation based on photonic integrated circuits (PICs) [2,3]. Dense photonic integration allows the fundamental block/device/module(s) of the network node element to be characterized by reduced cost, power consumption and footprint. The modular architecture allows suitably sizing the (S)-BVT according to the network/node needs. A programmable modular photonic transceiver enables advanced features, such as the slice-ability, multi-flow operation and the (re)-configuration of a set of parameters, specifically related to the adopted digital signal processing (DSP), optoelectronic frontend design, photonic technologies and devices [4]. Suitable transceiver architectures and solutions should be investigated, according to the technology options and the network segment requirements. For example, photonic technologies targeting low-cost and low power consumption are mandatory for cost-sensitive applications, especially for targeting the access and metro/aggregation segments. On the other hand, particularly when approaching the regional/core segment, it is of prominent relevance achieving ultimate performance in terms of capacity/bandwidth, serving a very high number of sites over an extended reach.

In this paper, different options for the S-BVT architecture/implementation are described, discussing the implications, advantages and limitations of the technological choices, with special focus on the programmability, flexibility and softwarization of the optical transmission system.

\section{SDN-enabled adaptive transmission systems: photonic technologies, programmability and softwarization}

Figure 1 shows the evolutionary network scenario, as described in Sec.1. The adoption of S-BVTs enables/facilitates a (soft) migration towards a more flexible paradigm and elastic optical networks (EONs) [4]. A transparent service delivery at variable data rates could be envisioned across the access and metro segments of an SDN optical network [5]. There, centralized S-BVT of high capacity (e.g. able to support ultrabroadband 5G services and high peak rate in a highly dynamic network scenario) are shared among distributed (S)-BVTs of lower capacity, closer to the enduser. The modular S-BVT adopts different photonic technologies/devices and optoelectronic frontends at the transmitter/receiver $(\mathrm{Tx} / \mathrm{Rx})$, suitably sizing the number of modules and/or module subset, which can be integrated on a same photonic platform as pluggable unit, for a scalable solution according to the node/segment needs.

Coherent and direct detection are technology options trading-off ultimate performance against reduced cost/complexity. Similarly, external modulation adopting e.g. Mach-Zehnder modulator(s) (MZM) and tunable laser source(s) (TLS) is a more costly solution, providing higher performance, than direct modulation. Photonic integrated 
multi-wavelength (multi- $\lambda$ ) locked optical combs, implemented using different technologies [6], represent a suitable alternative to independent TLS array for super-channel generation, at the expense of full tunability, programmability and flexibility $[3,4]$. Among direct modulated lasers (DMLs), vertical cavity surface emitting laser (VCSEL) is a promising technology for cost-sensitive applications, due to the lower cost than other common options (e.g. DFB lasers). Its potential at $1550 \mathrm{~nm}$ has been shown in the access and metro segments of (SDN) optical networks, with spectrum granularity even finer than EON [7]. Advanced functionalities can be enabled by the SDN controller, such as the mitigation of spectrum fragmentation without requiring a re-optimization of the entire network, thanks to the ability of spectral manipulation at sub-wavelength level and/or filling spectral gaps [4,7]. The former ability is particularly enabled by the adoption of multicarrier modulation technology and bit/power loading (BL/PL) algorithms at the DSP, which is (rate/distance) adaptive according to the traffic demand and the channel state information (CSI), retrieved at the (S)-BVRx (see e.g. Fig. 1 inset) [4]. The latter ability is eased by equipping the SBVTx with tunable VCSEL module(s) [7]. Super-channels and super-wavelength granularity can be enabled, optically processed and managed adopting either devices based on liquid crystal on silicon (LCoS) or planar ligthwave circuit (PLC) technology, trading tunability performance and cost $[4,8]$. The SDN controller suitably configures the specific element (device or module), setting each active slice/flow via the corresponding SDN agent that maps high level configuration commands to low level hardware-specific instructions. To target very high capacity per link, multiple dimensions can be exploited, integrating suitable devices/components, available and reconfigurable on-demand, in the S-BVT and/or network node. This includes polarization (PDM) and space (SDM) dimensions, in addition to full spectral manipulation with fine granularity [2]. Either fiber bundles or more advanced solutions like multi-core fibers (inset of Fig. 1) can be envisioned in an evolutionary softwarized scenario [9].

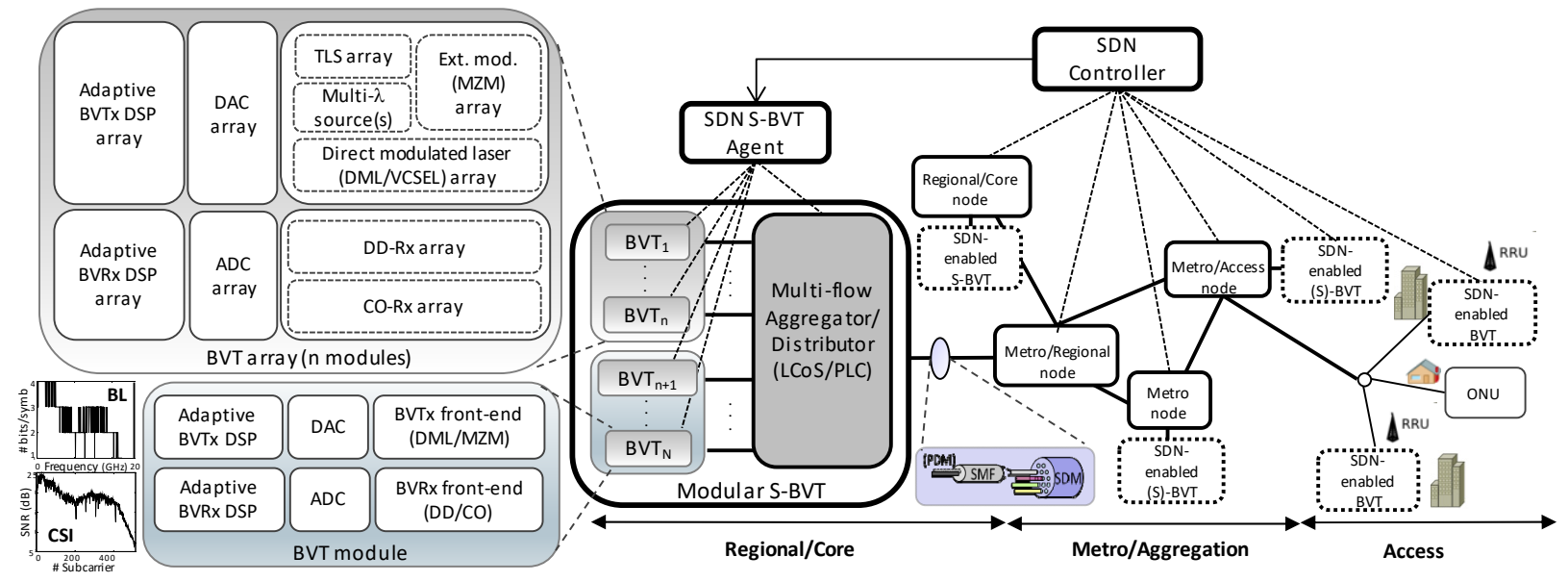

Fig. 1. Network scenario and programmable (SDN-enabled) transmission system. Alternative technologies for the modular S-BVT are indicated.

\section{Conclusions}

The challenges of hyperconnectivity can be addressed in an evolutionary optical network scenario with a suitable synergy of photonic and SDN technologies. Further efforts towards this goal should improve technology maturity and programmability, suitably integrating and softwarizing actual solutions to go beyond present limitations.

This work has been partially supported by the EU-H2020 PASSION Project (780326) and the Spanish DESTELLO project (TEC2015-69256-R).

\section{References}

[1] CISCO White Paper: "The Zettabyte Era: Trends and Analysis," June 2017.

[2] M. Svaluto Moreolo, J. M. Fabrega, and L. Nadal, "S-BVT for next-generation optical metro networks: benefits, design and key enabling technologies," in SPIE 10129, San Francisco, CA (USA), Jan. 2017.

[3] A. D'Errico, G. Contestabile, "Next generation terabit transponder," in OFC 2016, W4B.4, March 2016.

[4] M. Svaluto Moreolo et al., "SDN-Enabled Sliceable BVT Based on Multicarrier Technology for Multiflow Rate/Distance and Grid Adaptation,” IEEE/OSA J. Lightwave Technol. 34, 6, 1516-1522, March 2016.

[5] J. M. Fabrega, et al., "Experimental Validation of a Converged Metro Architecture for Transparent Mobile Front-/Back-Haul Traffic Delivery using SDN-enabled Sliceable Bitrate Variable Transceivers," IEEE/OSA J. Lightwave Technol. 36, 7, 1429-1434, Apr. 2018.

[6] M. Imran, et al., "Techno-economic analysis of carrier sources in slice-able bandwidth variable transponders," in ECOC 2016, Sept. 2016.

[7] M. Svaluto Moreolo, et al., "Modular SDN-enabled S-BVT Adopting Widely Tunable MEMS VCSEL for Flexible/Elastic Optical Metro Networks," in OFC 2018, paper M1A.7, S. Diego, CA (USA), March 2018.

[8] L. Nadal, et al., "Hybrid Electro-Optical MCM as Multi-Flow Generation Enabler for Elastic Optical Networks," in CLEO/Europe 2017.

[9] R. Muñoz et al., "SDN-enabled sliceable multi-dimensional (spectral and spatial) transceiver controlled with YANG/NETCONF," in OFC 2018, paper M2A.5, March 2018. 